Unfallchirurg 2007 $\cdot 110: 691-700$

DOI 10.1007/s00113-007-1310-y

Online publiziert: 9 . August 2007

(c) Springer Medizin Verlag 2007

\section{Rubrikherausgeber}

D. Nast-Kolb, Essen

T. Mittlmeier, Rostock

H. Reilmann, Braunschweig

\author{
V. Valderrabano ${ }^{1} \cdot$ M. Wiewiorski ${ }^{1}$ A. Frigg ${ }^{1} \cdot$ B. Hintermann ${ }^{2} \cdot$ A. Leumann ${ }^{1}$ \\ ${ }^{1}$ Orthopädische Universitätsklinik, Behandlungszentrum Bewegungsapparat, \\ Universitätsspital Basel \\ ${ }^{2}$ Orthopädische Klinik, Kantonsspital, Liestal
}

\title{
Chronische Instabilität des oberen Sprunggelenks
}

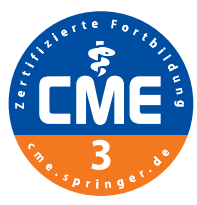

CME.springer.de - Zertifizierte Fortbildung für Kliniker und niedergelassene Ärzte

Die CME-Teilnahme an diesem Fortbildungsbeitrag erfolgt online auf CME.springer.de und ist Bestandteil des Individualabonnements dieser Zeitschrift. Abonnenten können somit ohne zusätzliche Kosten teilnehmen.

Unabhängig von einem Zeitschriftenabonnement ermöglichen Ihnen CME.Tickets die Teilnahme an allen CME-Beiträgen auf CME.springer.de. Weitere Informationen zu CME.Tickets finden Sie auf CME.springer.de.

\section{Registrierung/Anmeldung}

Haben Sie sich bereits mit Ihrer Abonnementnummer bei CME.springer.de registriert? Dann genügt zur Anmeldung und Teilnahme die Angabe Ihrer persönlichen Zugangsdaten. Zur erstmaligen Registrierung folgen Sie bitte den Hinweisen auf CME.springer.de.

\section{Zertifizierte Qualität}

Diese Fortbildungseinheit ist mit 3 CME-Punkten zertifiziert von der Landesärztekammer Hessen und der Nordrheinischen Akademie für Ärztliche Fort- und Weiterbildung und damit auch für andere Ärztekammern anerkennungsfähig. Folgende Maßnahmen dienen der Qualitätssicherung aller Fortbildungseinheiten auf CME.springer.de: Langfristige Themenplanung durch erfahrene Herausgeber, renommierte Autoren, unabhängiger Begutachtungsprozess, Erstellung der CME-Fragen nach Empfehlung des IMPP mit Vorabtestung durch ein ausgewähltes Board von Fachärzten.

Für Fragen und Anmerkungen stehen wir Ihnen jederzeit zur Verfügung:

Springer Medizin Verlag GmbH

Fachzeitschriften Medizin/Psychologie

CME-Helpdesk, Tiergartenstraße 17

69121 Heidelberg

E-Mail: cme@springer.com

CME.springer.de

\section{Zusammenfassung}

Die chronische Sprunggelenkinstabilität ist eine typische Pathologie im Sport und entwickelt sich bei 20 bis 40\% der Sportler nach erlittener akuter Sprunggelenkdistorsion. Man unterscheidet zwischen einer lateralen und medialen Sprunggelenkinstabilität, wobei die Kombination beider als Rotationsinstabilität des oberen Sprunggelenks bezeichnet wird. Des Weiteren kann pathophysiologisch eine mechanische oder funktionelle Sprunggelenkinstabilität unterschieden werden, die sich durch eine strukturelle Bandläsion bzw. durch eine Schwächung der neuromuskulären Kontrolle manifestiert. Durch die erschwerte Diagnose und eine komplexe Therapie, die oft eine operative Rekonstruktion bedingt, stellt die chronische Sprunggelenkinstabilität für den behandelnden Arzt eine vielschichtige Entität dar. Die vorliegende Übersichtsarbeit erörtert Pathomechanismen, Möglichkeiten der Diagnostik, Indikationen zur konservativen und operativen Therapie sowie potenzielle Langzeitschäden wie die posttraumatisch-ligamentäre Sprunggelenksarthrose.

\section{Schlüsselwörter}

Ligament $\cdot$ Instabilität $\cdot$ Sprunggelenk $\cdot$ Sport

\section{Chronic ankle instability}

\section{Abstract}

Chronic ankle instability represents a typical sports injury. After an acute ankle sprain $20-40 \%$ of the injured develop chronic ankle instability. From an orthopaedic point of view chronic ankle instability can be subdivided into lateral and medial instability or a combination of both, the socalled rotational ankle instability. From a pathophysiological point of view, chronic ankle instability can be either mechanical with a structural ligament lesion or functional with loss of neuromuscular control. For the physician chronic ankle instability is a difficult entity as the diagnosis is usually complex and the therapy often surgical. This review on chronic ankle instability deals with the pathomechanisms, diagnostics, indications for conservative and surgical treatments, and possible long-term sequelae, such as ligamentous osteoarthritis.

\section{Keywords}

Ligament · Instability · Ankle · Sports 


\section{Akute Sprunggelenkdistorsionen}

Eine chronische Instabilität weist typische Symptome auf

\section{- Risikosportarten}

Zur Gruppe mit dem höchsten Distorsionsrisiko zählen Basketball, Handball, Fußball, American Football, Rugby und Kunstturnen

Eine bereits erlittene Distorsion ist der größte Risikofaktor für eine weitere
Die vergangenen Jahre haben eine signifikante Zunahme von muskuloligamentären Verletzungen der unteren Extremität gezeigt. Mit 40\% aller Sportverletzungen zählen $>$ akute Sprunggelenkdistorsionen zu den häufigsten Verletzungen in der Sporttraumatologie [1]. Der häufigste Pathomechanismus aller Distorsionen ist mit $85 \%$ das Supinationstrauma, das zumeist eine Läsion des lateralen Bandapparates verursacht. Etwa 10 bis 15\% der Distorsionen durch ein Pronationstrauma bedingen meist mediale Bandläsionen [2]. Bei akuten Distorsionen können die tibio-fibulare Syndesmose, der subtalare Bandapparat sowie auch weitere osteochondrale oder Weichteilstrukturen (z. B. Sehnen, Nerven, Gefäße) begleitend betroffen sein.

Etwa 20 bis 40\% der Patienten entwickeln im späteren Verlauf nach einer akuten Distorsion eine chronische Sprunggelenkinstabilität [2]. Eine chronische Instabilität weist typische Symptome auf, z. B.:

- rezidivierende Distorsionen,

- Schmerzen im Bereich des Sprunggelenks,

- subjektives Instabilitätsgefühl (,giving way“),

- Schwierigkeiten beim Gehen und Rennen auf unebenem Gelände.

2 potenzielle Pathomechanismen werden diskutiert:

- mechanische Instabilität in Form einer strukturellen posttraumatischen Bandinstabilität,

- funktionelle Instabilität mit reduzierter Gelenkkontrolle durch eine Verschlechterung der propriozeptiven neuromuskulären Reaktionskette.

Insbesondere die subjektive Instabilität soll Ausdruck einer funktionellen Instabilität sein [3].

- Risikosportarten wie Fußball, Basketball, Handball, Judo, Orientierungslauf oder Tanz gefährden den Sportler enorm und sind mit Distorsionsraten von bis zu 80\% assoziiert [4]. Die Gefahr der Entwicklung einer chronischen Sprunggelenkinstabilität ist bei bereits erlittener Erstsprunggelenkdistorsion sowie ungenügender sportmedizinischen Primärversorgung signifikant erhöht.

Die vorliegende Übersichtsarbeit soll dem Leser einen Überblick zur chronischen Sprunggelenkinstabilität geben und sich mit wichtigen Fragen bezüglich der chronischen Sprunggelenkinstabilität im Freizeit- und Spitzensport befassen: Worin liegt die Relevanz der chronischen Sprunggelenkinstabilität im Bereich des Freizeit- und Wettkampfsports? Was sind die diagnostischen und therapeutischen Möglichkeiten eines behandelnden Arztes? Welche Gefahren und Komplikationen lauern auf Athleten mit einer chronischen Sprunggelenkinstabilität?

\section{Epidemiologie}

Eine sehr hohe Rate an akuten Distorsionen wurde bei bestimmten Sportarten wie Orientierungslauf (84\%), Basketball (70\%), Fußball (67\%) und Tanz (28\%) beschrieben. Zahlreiche Publikationen sind zu diesen einzelnen Sportarten erschienen. Halasi et al. [5] publizierten eine neue Zusammenstellung, welche das Distorsionsrisiko in einem , ankle activity score" zusammenzufassen versucht. $\mathrm{Zu}$ der Gruppe mit dem höchsten Distorsionsrisiko (Risikofaktor 9 von 10) zählen dabei Basketball, Handball, Fußball, American Football, Rugby und Kunstturnen. Distorsionsfrequenzen von bis zu 2,1 Verletzungen pro 1.000 Stunden Sportexposition wurden angegeben.

Zur Prävalenz der chronischen Instabilität des oberen Sprunggelenks liegen sehr wenige Daten vor. In einer Studie von Knobloch et al. wurde diese beim Orientierungslauf mit $60 \%$ angegeben und lag im Vergleich zu Leichtathletik und Bobsport signifikant höher [9]. Leumann et al. [6] konnten sogar bei 73\% der untersuchten Orientierungsläufer eine chronische Sprunggelenkinstabilität nachweisen.

Bei 380 Sportlern Hongkongs fanden Yeung et al. [7] in 73\% der Fälle rekurrente Distorsionen, wovon 59\% (entsprechend 43\% aller Athleten) über prolongiert persistierende Beschwerden und Einschränkungen berichtet haben.

Die Risikofaktoren der akuten Distorsionen wurden eingehend analysiert und in intrinsische und extrinsische Faktoren unterteilt. Es ist weitgehend unklar, welche dieser Faktoren für die Entstehung einer chronischen Instabilität qualifizieren. Es ist unbestritten, dass der größte Risikofaktor, eine weitere Distorsion zu erleiden, die bereits erlittene Distorsion ist. 
Abb. 1 - Anatomisch-orthopädische Einteilung der chronischen Instabilität des oberen Sprunggelenks: Die Rotationsinstabilität des oberen Sprunggelenks ist eine kombinierte laterale und mediale Instabilität

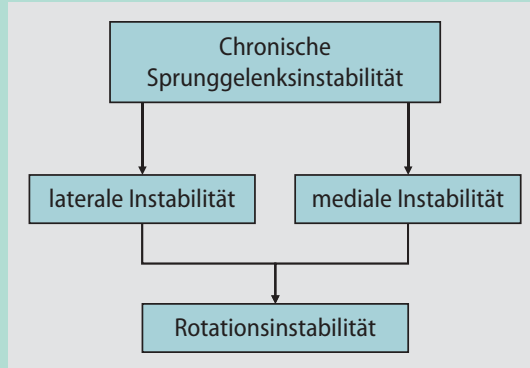

Abb. 2 Pathomechanistische Einteilung der chronischen Instabilität des oberen Sprunggelenks: Die chronische Sprunggelenkinstabilität wird in eine mechanische und eine funktionelle Instabilität unterteilt, die sich gegenseitig potenzieren können

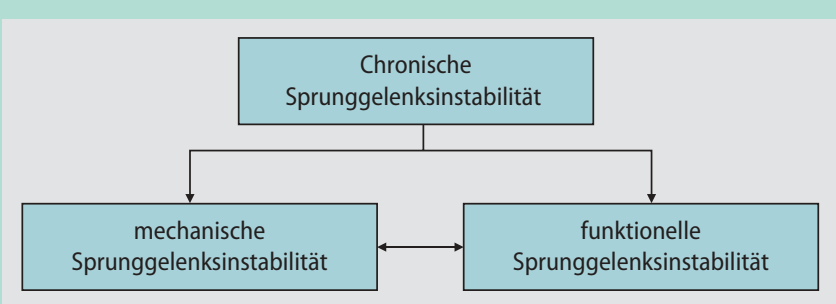

\section{Instabilitätstypen und Pathomechanismen}

Der Bandapparat des Sprunggelenkes besteht lateralseits aus dem Ligamentum fibulotalare anterius (LFTA), dem Ligamentum fibulocalcaneare (LFC) und dem Ligamentum fibulotalare posterius (LFTP). Funktionell muss ebenso das laterale Ligamentum talocalcaneare dazugezählt werden. Während das LFTA der Inversion vor allem in Plantarflexion und neutraler Flexionsstellung entgegenwirkt, kontrollieren das LFC und LFTP die Inversion in der Dorsalextension. Medialseits wird die ligamentäre Stabilität durch das Ligamentum deltoideum gewährleistet, welches aus oberflächlichen (v. a. die Talusabduktion limitierenden Fasern) und tiefen (v. a die Tibiaaußenrotation limitierenden Fasern) Anteilen besteht und direkt mit dem für das mediale Fußlängsgewölbe entscheidenden Springligament kommuniziert [8]. Liegt eine traumatische Insuffizienz oder vollständige Ruptur eines oder mehrerer Bänder vor, so spricht man in der anatomisch-orthopädischen Einteilung von einer $>$ lateralen bzw. medialen Instabilität des oberen Sprunggelenks [3]. Liegt eine kombinierte laterale und mediale Instabilität vor, so spricht man von einer $>$ Rotationsinstabilität des oberen Sprunggelenks ( $\mathbf{A b b}$. 1). In-vitro-Studien haben gezeigt, dass mit der Durchtrennung von LFTA und LFC zwar die laterale Instabilität („talar tilt“) deutlich zunimmt, es jedoch bei einer zusätzlichen Durchtrennung des Lig. deltoideum zu einer Zunahme der Rotation im oberen Sprunggelenk kommt, was die normalerweise durch das untere Sprunggelenk ausgelöste Tibiaaußenrotation als Folge einer Kalkaneusinversion verstärkt [9]. Wie bei den akuten Distorsionen ist auch bei der chronischen Sprunggelenkinstabilität mehrheitlich der laterale Bandapparat betroffen. Die eigene klinische Erfahrung zeigt jedoch, dass die Diagnose einer medialen Sprunggelenkinstabilität schwierig ist und somit in orthopädischen Fachkreisen oftmals verpasst wird.

Zusätzlich zur anatomisch-orthopädischen Klassifikation wird die chronische Sprunggelenkinstabilität in der pathomechanistischen Einteilung in eine mechanische und funktionelle Instabilität unterteilt (• Abb. 2). Die mechanische Instabilität gibt die strukturelle Insuffizienz und somit pathologische Aufklappbarkeit des Gelenks wieder, kann aber die subjektive Instabilitätssymptomatik nicht vollständig erklären. Pathomechanistisch wurde deshalb von Freeman der Begriff der funktionellen Instabilität geprägt [5]. Unter der funktionellen Stabilität versteht man in Analogie zum Knie die neuromuskuläre oder posturale Gelenkstabilisierung. Dies beinhaltet insgesamt eine Schwäche der posturalen Kontrolle sowie auch einzelner Komponenten (z. B. Propriozeption, Nervenleitung, zentrale Impulsverarbeitung, Kraft). Wichtig für den Athleten ist die Tatsache, dass mit der Ermüdung die funktionelle Stabilität deutlich abnimmt.

Es ist nach wie vor umstritten, ob es sich bei der funktionellen Instabilität um eine eigene Entität handelt oder ob sie eng mit der mechanischen Instabilität gekoppelt ist. Freeman fand in 40\%
LFC und LFTP kontrollieren die Inversion in der Dorsalextension

\section{Laterale bzw. mediale Instabilität Rotationsinstabilität}

Mit der Ermüdung nimmt die funktionelle Stabilität deutlich $a b$ 
Eine mechanische Instabilität ist im Spitzensport durchaus neuromuskulär kompensierbar

Die chronische Sprunggelenkinstabilität kann mit Begleit- oder Langzeitschäden vergesellschaftet sein

Chondrale Läsionen stellen häufige Begleitverletzungen chronischer Instabilität dar

\section{Kinematische Wirkung}

\section{Kraftrelaxationskurve}

\section{- Spannungs-Dehnungs- Verhalten}

Goldstandard der Instabilitätsdiagnostik stellt die invasive diagnostische Arthroskopie dar

Der Kliniker muss und soll primär auf Anamnese und klinische Untersuchung zurückgreifen der Fälle nach akuter Distorsion eine persistierende funktionelle Instabilität [5]. Tropp konnte diese Befunde bei Fußballspielern nicht nachvollziehen, fand aber, dass funktionell instabile Athleten ein signifikant größeres Risiko hatten, erneute Distorsionen zu erleiden [4]. Hubbard et al. [10] fanden bei subjektiver, unilateraler funktioneller Instabilität eine signifikant erhöhte mechanische Instabilität in sagittaler Richtung von 19,8 vs. 18,3 mm anteriorer Verschiebbarkeit. Hertel et al. [11] beschreiben den Zusammenhang von funktioneller und mechanischer Instabilität als Kontinuum mit einem stufenlosen Übergang. Während bislang alle Studien bei chronischer Sprunggelenkinstabilität eine funktionelle Schwäche zeigten, konnten im Gegensatz dazu Leumann et al. [10] an einer Gruppe von Eliteorientierungsläufern zeigen, dass diese trotz mechanischer Instabilität ein gesteigertes neuromuskuläres Stabilisationspotenzial haben können. Es ist deshalb davon auszugehen, dass eine mechanische Instabilität im Spitzensport durchaus neuromuskulär kompensierbar ist. Die Autoren hypothetisieren deshalb, dass die Sprunggelenke sich vor Langzeitschäden einer chronischen Instabilität durch ein gesteigertes neuromuskuläres Potenzial schützen können. Die chronische Sprunggelenkinstabilität kann mit Begleit- oder Langzeitschäden vergesellschaftet sein. Arthroskopische Untersuchungen von Hintermann et al. [12] an symptomatischen chronisch instabilen Sprunggelenken haben gezeigt, dass mit 54\% talaren, vor allem medialseits gelegenen, und 23\% kruralen Knorpelschäden chondrale Läsionen häufige Begleitverletzungen chronischer Instabilität darstellen. Schwere Knorpelschäden lagen insbesondere bei Rotationsinstabilität vor. Der Zusammenhang von chronischer Sprunggelenksinstabilität und chondralen Läsionen liegt somit auf der Hand. Der genaue Pathomechanismus ist jedoch ebenso wie eine erfolgreiche Langzeittherapie immer noch weitgehend unklar.

\section{Biomechanik der Bänder am oberen Sprunggelenk}

Die breiten Ligamente (Lig. fibulotalare anterius, LFTA; Lig. fibulocalcaneare, LFC; Lig. fibulotalare posterius, LFTP; Lig. deltoideus) und die großflächigen Insertionsstellen führen dazu, dass die - kinematische Wirkung der Bänder im Bewegungsablauf nicht konstant ist. Das LFTA wirkt wegen seiner Geometrie und anatomischen Ausrichtung entgegen einer anterioren Dislokation respektive Luxation und stabilisiert das Gelenk vor allem in Plantarflexion und zusätzlicher Supination. Das LFC zeigt seine größte Spannung und damit Stabilisation in Dorsalextension und Supination. Das LFTP wirkt stabilisierend in Dorsalextension, kombiniert mit Valgusaußenrotationsstress. Der Deltoid-Ligament-Komplex stabilisiert Pronation und Eversion bzw. Abduktion; sein posteriorer Teil insbesondere in Dorsalextension, der anteriore Teil in Plantarflexion. Dieser Effekt wird durch die intraligamentäre fächerförmige Struktur der Ligamente erklärt.

Die Kraftrelaxationskurve von Bändern zeigt, dass Bänder auf einen plötzlichen Spannungsanstieg mit einer Widerstandskraft reagieren, die nach einem initialen Kraft-Peak kontinuierlich abnimmt. Dies wird ebenso wie das nichtlineare $>$ Spannungs-Dehnungs-Verhalten durch die molekulare Zusammensetzung aus fibrillärem Kollagen (70-80\% der Trockenmasse), Elastin (10-15\% der Trockenmasse), Proteoglykanen (1-3\% der Trockenmasse) und Wasser (66\% des Feuchtgewichts) bewirkt, welche dem Ligamentgewebe sein biomechanisch viskoelastisches Verhalten verleiht [13].

\section{Diagnostik}

Eine chronische Instabilität kann über verschiedene Methoden (klinisch, radiologisch, invasivdiagnostisch) messbar gemacht werden (• Tab. 1).

Goldstandard in der Instabilitätsdiagnostik stellt die invasive diagnostische Arthroskopie des oberen Sprunggelenks dar ([4, 14]; - Abb. 3). Damit können ligamentäre, osteochondrale und degenerative Veränderungen erfasst sowie die Bandstabilität unter direkter Sicht geprüft werden. In der Praxis des Orthopäden und in der präoperativen Abklärung steht dieses invasivdiagnostische Werkzeug jedoch nicht zur Verfügung. Der Kliniker muss und soll deshalb primär auf die Anamnese und die klinische Untersuchung zurückgreifen.

In der Anamnese ist akribisch einzugehen auf:

- Schmerzen,

- Distorsionshäufigkeit,

- Unfallmechanismus,

- subjektives Instabilitätsgefühl, welches häufig als „Giving-way“-Gefühl beschrieben wird [5]. 
Tab. 1 Diagnostik der Sprunggelenkinstabilität

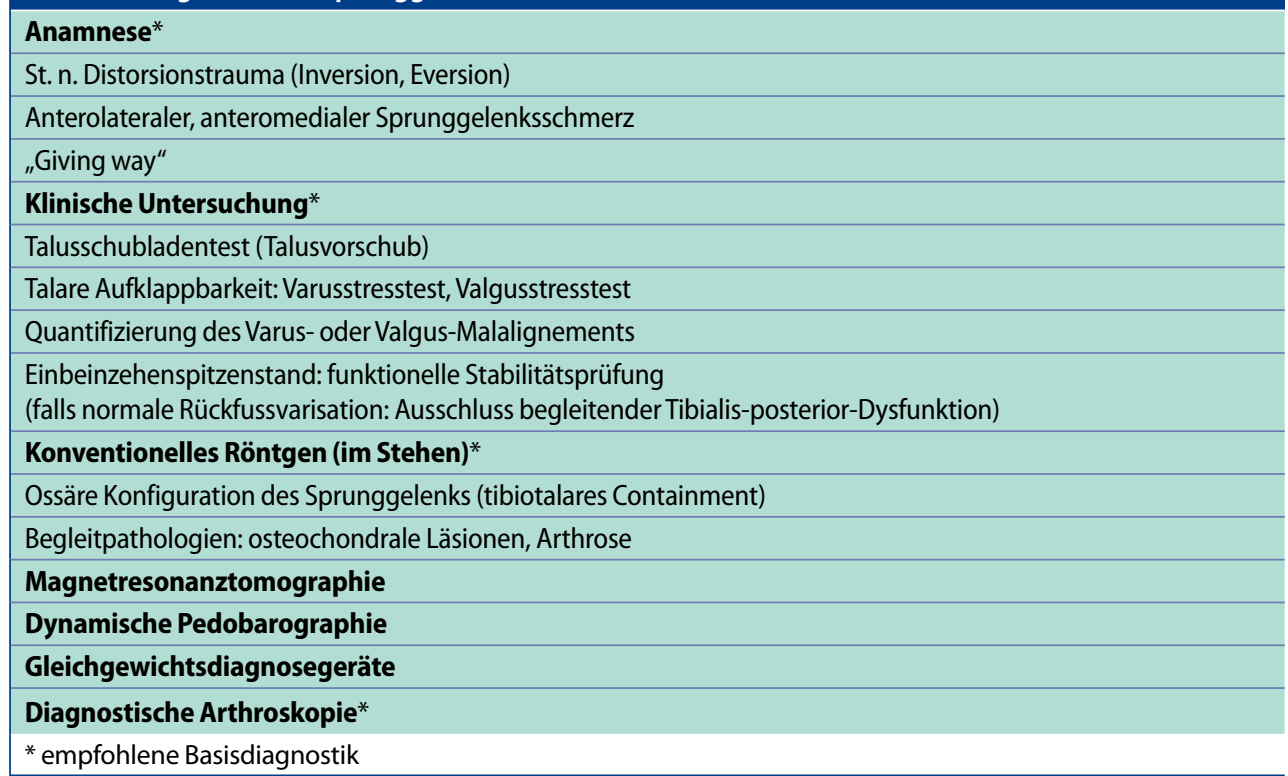

Auch systemische Erkrankungen mit Einfluss auf die ligamentäre Stabilität des Sprunggelenks (z. B. Ehler-Danlos-Syndrom, Marfan-Syndrom etc.) sind anamnestisch zu erfragen.

Die klinische Untersuchung erfasst wichtige Pathologien mittels Inspektion und Palpation der Bandstrukturen, Sehnen und Sehnenansatzpunkte. Für die laterale Sprunggelenkinstabilität ist eine Druckdolenz am gemeinsamen fibulären Ursprung des LFTA und LFC sowie am Sinus tarsi typisch, für die mediale Instabilität eine Druckdolenz im Bereich des ventralen Deltoids und des Springligaments. Zur Erfassung der Bandinstabilität wird mit dem $>$ anterioren Drawer-Test (Schubladentest; [15]) die Talusverschieblichkeit nach anterior und damit insbesondere das LFTA geprüft, mit dem - lateralen Talar-Tilt-Test (Inversionsstresstest, lateraler Aufklappbarkeitstest) das LFC und mit dem - medialen Talar-Tilt-Test (Eversionsstresstest, medialer Aufklappbarkeitstest) der mediale Bandapparat, also das Deltoid mit seinen tiefen und oberflächlichen Anteilen. Wichtig ist hierbei insbesondere der Seitenvergleich mit der nicht betroffenen Seite, um eine konstitutive Hyperlaxität erfassen bzw. ausschließen zu können.

Eine mechanische Bandinsuffizienz kann durch die periartikuläre Muskulatur teilweise oder gänzlich kompensiert werden. Kommt es jedoch im Verlauf zu einer Überlastung der stabilisierenden Muskulatur (medialseits der Tibialis posterior und lateralseits der Peronealmuskulatur) wird die chronische Instabilität symptomatisch. In diesem Zusammenhang muss dringendst von Steroidinfiltrationen von Sehnen oder peritendinösem Gewebe abgeraten werden. Bereits einmalige Kortisoninjektionen können zur Schwächung der Sehne und zu degenerativen Rupturen führen.

Weiter ist die Beurteilung des $>$ Rückfuß-Alignements und des Fußgewölbes wichtig. Eine chronische mediale Sprunggelenkinstabilität korreliert häufig mit einer Valgusdeformität des Rückfußes, eine laterale mit einer Varusdeformität. Die Differenzialdiagnose zwischen der medialen Instabilität und der Tibialis-posterior-Insuffizienz ist entscheidend. Im Stehen kann nämlich oft eine Kompensation der medialen Sprungelenkinstabilität durch die Aktivierung des Tibialis-posterior-Muskels beobachtet werden. Mit der Varisierung der Ferse im Single-heel-rise-Test kann jedoch eine Tibialis-posterior-Insuffizienz klinisch ausgeschlossen werden. Weiter müssen Peronealpathologien oder Läsionen der tibiofibularen Syndesmose und des subtalaren und talonavikularen Gelenks erfasst werden, um sie in die Therapie miteinbeziehen zu können. Zur Beurteilung ossärer Veränderungen steht das konventionelle Röntgenbild zur Verfügung. Früher wurde versucht, die Bandinsuffizienz mittels Stressröntgen zu quantifizieren. Eine Metaanalyse von Frost und Amendola [16] konnte die Reliabilität des Stressröntgens widerlegen und auch die Gefahr der Provokation einer zusätzlichen Verletzung unter Anästhesie aufzeigen. Daraus folgend kann die Autorengruppe Stressröntgenbilder oder gehaltene Aufnahmen nicht mehr unterstützen. Zur Beurteilung der Gelenkkongruität ist jedoch eine gewichtsbelastete, stehende Röntgenaufnahme ohne Stress unabdingbar. Diese dient dem Ausschluss knöcherner Pathologien sowie der Analyse der ossären Konfiguration des

\section{Anteriorer Drawer-Test}

- Lateraler Talar-Tilt-Test - Medialer Talar-Tilt-Test

Von Steroidinfiltrationen von Sehnen oder peritendinösem Gewebe wird dringend abgeraten

\section{- Rückfuß-Alignement}

Eine chronische mediale Sprunggelenkinstabilität korreliert häufig mit einer Valgusdeformität des Rückfußes, eine laterale mit einer Varusdeformität

Zur Beurteilung ossärer Veränderungen steht das konventionelle Röntgenbild zur Verfügung 


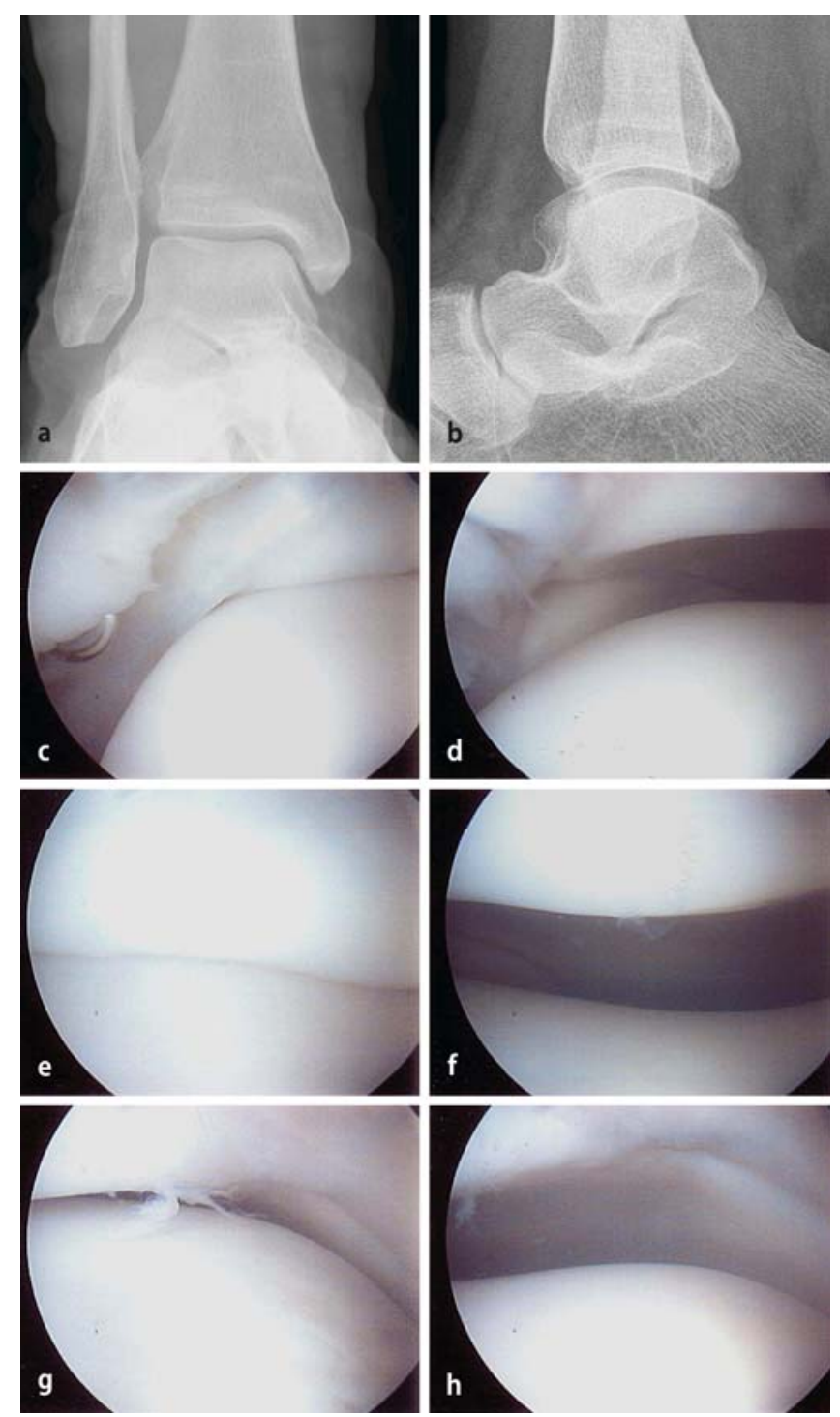

Abb. $3 \varangle$ Fall einer chronischen Rotationsinstabilität des oberen Sprunggelenks: anteroposteriores (a) und laterales (b) Röntgenbild mit hohem ossären Taluskörper und typischerweise reduzierter tibialer Abdeckung des Talus (Containment); in der Arthroskopie vor ligamentärer Rekonstruktion zeigt sich eine kombinierte laterale $(\mathbf{c}, \mathbf{d})$ und mediale $(\mathbf{g}, \mathbf{h})$ Instabilität (Rotationsinstabilität); zusätzlich Sicht auf zentralen Teil des Sprunggelenks $(\mathbf{e}, \mathbf{f})$

\section{Pathologisches tibiotalares Containment}

\author{
Wichtig ist auch der Ausschluss \\ von subtalaren oder tibiofibularen \\ Pathologien
}

Sprunggelenks. Ein Talus mit einem hohen Körper, Inkongruenzen am lateralen und medialen Maleolus sowie pathologische subtalare und talonavikulare Gelenkstellungen können auf eine Instabilität hinweisen. Zusätzlich konnten Frigg et al. im Vergleich zu gesunden Probanden eine signifikant kleinere Überdachung des Talus durch die Tibia und somit ein $>$ pathologisches tibiotalares Containment nachweisen [17].

Die Sonographie hat ihre Funktion in der Beurteilung von Begleitpathologien wie einer Peroneal- oder Tibialis-posterior-Sehnen-Entzündung. Alternativ und verbunden mit dem Vorteil von auf andere Strukturen erweiterten Fragestellungen kann eine Magnetresonanztomographie hinsichtlich der Beurteilung des Bandapparates, des Knorpelzustandes sowie der ossären Situation nützliche Auskünfte zum oberen Sprunggelenk geben. Wichtig ist auch der Ausschluss von subtalaren oder tibiofibularen Pathologien, welche mitursächlich für eine Instabilität sein können.

Die Diagnostik einer funktionellen Instabilität in einer orthopädischen Praxis ist schwierig. Geräte, welche die neuromuskuläre Stabilität untersuchen, können aus finanziellen Gründen oft nur durch größere sportorthopädische Zentren angeschafft werden. Mit diesen Geräten können pathologische neuromuskuläre Defizite im Seitenvergleich gemessen werden [4, 10]. In den letzten Jahren konnten damit folgende einzelne Variablen erarbeitet werden $[4,16]$ :

- Messung des Gleichgewichts,

- Messung der peronealen Reaktionszeit,

- Kraftmessungen einzelner Muskeln,

- Kraftvergleiche von Agonist und Antagonist am Sprunggelenk. 
Es mangelt jedoch noch an genügend validierten Normwerten. Messmethoden und publizierte Resultate sind sehr vielfältig, teilweise jedoch kontrovers und auf kleinen Fallzahlen basierend, sodass zum heutigen Zeitpunkt eine Empfehlung für den Orthopäden in der Praxis äußerst schwierig ist. Die Ganganalytik und die dynamische Baropedobarographie konnten bislang einige Hinweise zur Analyse der Pathomechanismen beitragen; für die diagnostische Anwendung fehlt jedoch die klinische Umsetzung.

Zur „hilfsmittelfreien“ Messung der funktionellen Stabilität sind verschiedene Tests beschrieben worden. Olmsted et al. [18] beobachteten beim $>$ „Star-excursion-balance“-Test signifikant schlechtere Resultate für Probanden mit einer unilateralen chronischen Sprunggelenksinstabilität mit 78,6 vs. $82,8 \mathrm{~cm}$ im Vergleich zur gematchten Kontrollgruppe und 78,6 vs. 81,2 cm im Vergleich zur gesunden Gegenseite. Dabei geht es darum, mit einem Bein im Zentrum eines 8-strahligen Sterns zu stehen und mit dem anderen Bein, immer das Gleichgewicht wahrend, auf jedem Strahl mit dem Großzeh den Boden so weit wie möglich vom Zentrum entfernt zu berühren. Kuni et al. [19] beschreiben den Einbeinstandtest, bei welchem im Einbeinstand mit geschlossenen Augen während einer Minute Bodenberührungen als Fehlerpunkte gezählt werden. Dabei fanden sich bei Tänzern signifikant bessere Werte im Vergleich zur Kontrollgruppe. Beide Tests haben jedoch ihre breite klinische Anwendbarkeit sowie eine Gradierung zur therapeutischen Implikation noch nicht zeigen können.

\section{Komplikationen/frühzeitige Sportinvalidität}

Neben den typischen Symptomen Sprunggelenkschmerz, chronisches Instabilitätsgefühl und rezidivierende Distorsionen, welche persistieren oder bis zu einer Belastungsunfähigkeit des Fußes führen können, steht insbesondere die raschere Entwicklung einer Arthrose des oberen Sprunggelenks im Mittelpunkt des Interesses. Zur Arthroserate bei chronischer Instabilität ohne ossäre Fraktur gibt es wenig publizierte Daten. Harrington et al. [20] haben 1979 erstmals einen Zusammenhang zwischen erhöhter Arthroseraten und chronischer Instabilität aufgezeigt. Valderrabano et al. konnten in ihrer OSG-Arthrose-Kohorte zeigen, dass 13\% aller posttraumatischen OSG-Arthrosen eine Folge ligamentärer Verletzungen waren [2]. Die durchschnittliche Latenzzeit zur Arthroseentwicklung betrug dabei 34 Jahre und war vom Instabilitätstyp abhängig.

Bei der Durchführung einer Arthroskopie bei chronischer Instabilität können osteochondrale Talusläsionen bis zu 66\% bei lateraler Instabilität und bis zu 98\% bei medialer Instabilität gefunden werden. [12] Der natürliche Verlauf dieser Läsionen ist nach wie vor unklar. So fanden Löfvenberg et al. [21] bei 37 Patienten mit 20-jähriger Instabilitätsanamnese in 16\% der Fälle Zeichen degenerativer Gelenkprozesse.

\section{Präventive und therapeutische Möglichkeiten}

Eine insuffiziente Therapie einer akuten Distorsion stellt den Ausgangspunkt für die Entstehung einer chronischen Instabilität dar. Insofern stellt die adäquate Therapie der akuten Sprunggelenkdistorsion die wichtigste Grundlage für die Prävention der chronischen Instabilität dar. Diese setzt sich nach einer initialen Abschwell- und Entlastungsphase aus einem frühfunktionellen propriozeptiv-neuromuskulären Stabilisationstraining und einer eventuell notwendigen Distorsionsprophylaxe mittels externer Stabilisation durch Taping, Bracing oder entsprechende Schuhversorgung zusammen.

Externe Stabilisatoren können die Distorsionsrate erfolgreich senken. Sie wirken einerseits über eine mechanische Einschränkung des Bewegungsausmaßes, andererseits aber auch über eine propriozeptive Stimulation.

Unumstritten wird als Therapie der chronischen Instabilität primär ein konservatives Vorgehen empfohlen. Durch propriozeptiv-neuromuskuläres Stabilisationstraining kann in vielen Fällen eine signifikant verbesserte funktionelle Stabilität erreicht werden (• Tab. 2). Verhagen et al. [22] konnten eine signifikante Senkung der Distorsionsrezidivrate bei regelmäßigem funktionellem Training bei Volleyballspielern zeigen.

Erst bei persistierenden Beschwerden nach missglücktem physiotherapeutischen Aufbau sollte auf eine chirurgische Therapie (Kombination von Arthroskopie und Stabilitätseingriff) zurückgegriffen werden. Als Stabilitätseingriff bietet sich hier die direkte Bandrekonstruktion nach

\section{Ganganalytik \\ Dynamische \\ Baropedobarographie}

„Star-excursion-balance“-Test

Einbeinstandtest

Arthrose

In der OSG-Arthrose-Kohorte waren $13 \%$ der posttraumatischen

OSG-Arthrosen eine Folge

ligamentärer Verletzungen

Wichtigste Grundlage für die Prävention einer chronischen Instabilität ist die adäquate Therapie der akuten Sprunggelenkdistorsion

Als Therapie der chronischen Instabilität wird primär ein konservatives Vorgehen empfohlen 


\begin{tabular}{|c|c|c|c|c|}
\hline & Phase 1 & Phase 2 & Phase 3 & Phase 4 \\
\hline \multirow[t]{2}{*}{$\begin{array}{l}\text { Propriozeption/ } \\
\text { Sensorik }\end{array}$} & $\begin{array}{l}\text { Innervationsübungen } \\
\text { (passiv und aktiv) }\end{array}$ & $\begin{array}{l}\text { Joint-Position- } \\
\text { Sense-Training, } \\
\text { integrative Übungen } \\
\text { mit Vorbereitung, } \\
\text { Gangschulung }\end{array}$ & $\begin{array}{l}\text { Integrative Übungen, } \\
\text { ohne Vorbereitung, } \\
\text { ohne Kontrolle, } \\
\text { Reaktions-/Gleich- } \\
\text { gewichtsübungen }\end{array}$ & $\begin{array}{l}\text { Rückführung } \\
\text { in den Sport }\end{array}$ \\
\hline & + & +++ & ++ & \\
\hline \multirow[t]{2}{*}{ Bewegungsumfang } & Passiv & Aktiv & Aktiv & $\begin{array}{l}\text { Rückführung } \\
\text { in den Sport }\end{array}$ \\
\hline & ++ & + & + & \\
\hline \multirow[t]{2}{*}{$\begin{array}{l}\text { Posturale Stabilität } \\
\text { (Feed-forward/ } \\
\text { Feed-backward) }\end{array}$} & $\begin{array}{l}\text { Stabilisierungs- } \\
\text { übungen } \\
\text { im Einbeinstand, } \\
\text { Zehenspitzenstand }\end{array}$ & $\begin{array}{l}\text { Stabilisierungs- } \\
\text { übungen auf } \\
\text { unebenem Boden, } \\
\text { "Wobble board“, } \\
\text { Gangschulung }\end{array}$ & $\begin{array}{l}\text { Stabilisierungs } \\
\text { übungen auf } \\
\text { unebenem Boden } \\
\text { mit zusätzlichen } \\
\text { koordinativ-kogni- } \\
\text { tiven Anforderungen, } \\
\text { geschlossenen } \\
\text { Augen }\end{array}$ & $\begin{array}{l}\text { Rückführung } \\
\text { in den Sport }\end{array}$ \\
\hline & ++ & +++ & +++ & \\
\hline \multirow[t]{2}{*}{ Kraft/Motorik } & $\begin{array}{l}\text { Aktiv innervierend, } \\
\text { wenig belastend }\end{array}$ & $\begin{array}{l}\text { Aktiv mit eigenem } \\
\text { Körpergewicht } \\
\text { (v. a. Inversion/ } \\
\text { Eversion und Exten- } \\
\text { sion/Flexion) }\end{array}$ & $\begin{array}{l}\text { Aktiv forciert, } \\
\text { mit Gewichten, } \\
\text { Hilfsmitteln } \\
\text { (Thera-Band) }\end{array}$ & $\begin{array}{l}\text { Rückführung } \\
\text { in den Sport }\end{array}$ \\
\hline & + & ++ & +++ & \\
\hline \multicolumn{5}{|c|}{$\begin{array}{l}\text { Einteilung der Trainingseinheiten in die verschiedenen Aspekte von propriozeptiv-neuromuskulärem Training } \\
\text { entsprechend der Rehabilitationsphasen. Die Gewichtung der einzelnen Ansätze ist in "wichtig“ (+), ,,sehr } \\
\text { wichtig“ (++) und „am wichtigsten“ (+++) unterteilt. Die Rehabilitationsansätze in anderer Gewichtung gelten } \\
\text { auch für das Aufbautraining nach operativer Stabilisation. Phase } 4 \text { wird als Rückführung in den (Spitzen-)Sport } \\
\text { verstanden. Sie beinhaltet die Integration aller Elemente und ist bei erfolgreicher Stabilisierung und Rezidiv- } \\
\text { prophylaxe auch in unkontrollierbaren Situationen erreicht }\end{array}$} \\
\hline
\end{tabular}

\section{Anatomische Sehnenrekonstruktion}

\section{Dwyer-Osteotomie}

Entscheidend für den Therapieerfolg ist primär der direkte Kontakt zum Athleten
Brostrom-Gould [23] oder bei fehlendem In-situ-Bandmaterial die anatomische Sehnenrekonstruktion z. B. mittels Plantaris-longus-Sehne an [24]. Nicht mehr empfohlen werden nichtanatomische Tenodesetechniken wie die nach Evans oder Watson-Jones, da diese einen beträchtlichen Prozentsatz an Langzeitversagen zeigten, verursacht durch biomechanisch unphysiologische Gelenks- und Knorpelbelastungen, die zu einer Arthrosebildung beitragen können. Krips et al. [25] fanden in einer Multizenterstudie bei Tenodesen signifikant höhere Arthrosezeichen, ein eingeschränktes Bewegungsausmaß und ein schlechteres subjektives Resultat nach 2 bis 10 Jahren Nachuntersuchungszeit.

Bevor eine Bandstabilisation in Angriff genommen werden kann, sollten in jedem Fall Begleitpathologien (z. B. Tibialis-posterior-Insuffizienz, Peronalsehnenluxationen, subtalare Instabilitäten) ausgeschlossen werden und eine Analyse des Rückfuß-Alignements vorgenommen und gegebenenfalls korrigiert werden. So kann z. B. mit einer Dwyer-Osteotomie eine chronische OSGInstabilität mit Varus-Rückfuß-Deformität erfolgreich behandelt werden. Entscheidend für den Erfolg einer Therapie dürfte jedoch primär der direkte Kontakt zum Athleten sein. Der Leistungssportler steht in einem starken Spannungsfeld aus eigenen Zielen sowie Erwartungen von Trainern, Team und Sponsoren, welche oft wenig Rücksicht auf die Verletzung eines Athleten und auf medizinische Aspekte von Arzt und Physiotherapeut nehmen. Die Autoren sind der Überzeugung, dass es entscheidend ist, den Patienten eng zu führen, indem unrealistische Versprechungen vermieden werden, und unter Einbeziehen von Saisonplanung, Trainer und Umfeld mit dem Athleten von Beginn an schrittweise einen Weg zur möglichst schnellen Rückkehr in den Wettkampfsport zu planen. 


\section{Fazit für die Praxis}

Das biomechanische Konzept der chronischen Instabilität am oberen Sprunggelenk hat gezeigt, dass alle gelenkbildenden Strukturen ursächlich oder sekundär beteiligt sein können. Die chronische Sprunggelenkinstabilität kann sich anatomisch durch eine laterale, mediale oder Rotationsinstabilität manifestieren, pathomechanistisch durch eine mechanische und/oder funktionelle Instabilität. Diese komplexe Situation verlangt vom Arzt eine systematische und breite klinische und bildgebende Diagnostik. Bei der heutigen Datenlage ist davon auszugehen, dass im Sport mit spezifischem Aufbautraining eine mechanische Instabilität funktionell teilweise oder gänzlich kompensiert werden kann. Darum soll zu Beginn der Therapie immer der Versuch einer konservativen Maßnahme stehen. Ist diese ausgeschöpft, muss gegebenenfalls die Indikation zur operativen Therapie mittels Arthroskopie und Bandrekonstruktion gestellt werden. Die Datenlage bezüglich operativer Langzeitresultate wie auch bezüglich Spätschäden ist jedoch nach wie vor sehr spärlich. Zu diesen Aspekten sind weitere Studien und neue Ansätze erforderlich.

\section{Korrespondenzadresse}

PD Dr. Dr. V. Valderrabano

Orthopädische Universitätsklinik, Behandlungszentrum Bewegungsapparat, Universitätsspital Basel Spitalstraße 21, CH-4031 Basel

Schweiz

vvalderrabano@uhbs.ch

Interessenkonflikt. Der korrespondierende Autor gibt an, dass kein Interessenkonflikt besteht.

\section{Literatur}

1. Valderrabano V, Hintermann B, Horisberger $\mathrm{M}$ et al. (2006) Ligamentous posttraumatic ankle osteoarthritis. Am J Sports Med 34: 612-620

2. Hintermann B, Valderrabano V, Boss A et al. (2004) Medial ankle instability: an exploratory, prospective study of fifty-two cases. Am J Sports Med 32: 183-190

3. Freeman M, Dean M, Hanman W (1965) The etiology and prevention of functional instability of the foot. J Bone Joint Surg Br 47: 678-685

4. Valderrabano V, Hintermann B (2005) Diagnostik und Therapie der medialen Sprunggelenksinstabilität. Arthroskopie 18: 112-118

5. Halasi T, Kynsburg A, Tallay A et al. (2004) Development of a new activity score for the evaluation of ankle instability. Am J Sports Med 32: 899 908

6. Leumann A, Valderrabano V, Marti B et al. (2007) Chronic ankle instability in the Swiss orienteering national team. Scand J Med Sci Sports (submitted)

7. Yeung MS, Chan KM, So CH et al. (1994) An epidemiological survey on ankle sprain. Br J Sports Med 28 : 112-116

8. Boss AP, Hintermann B (2002) Anatomical study of the medial ankle ligament complex. Foot Ankle Int 23: 547-553

9. Cass JR, Settles H (1994) Ankle instability: in vitro kinematics in response to axial load. Foot Ankle Int 15: 134140
10. Hubbard TJ, Kaminski TW, Van der Griend RA et al. (2004) Quantitative assessment of mechanical laxity in the functionally unstable ankle. Med Sci Sports Exerc 36: 760-766

11. Hertel J (2002) Functional anatomy, pathomechanics, and pathophysiology of lateral ankle instability. J Athl Train 37: 364-375

12. Hintermann B, Boss A, Schafer D (2002) Arthroscopic findings in patients with chronic ankle instability. Am J Sports Med 30: 402-409

13. Funk JR, Hall GW, Crandall JR et al. (2000) Linear and quasi-linear viscoelastic characterization of ankle ligaments. J Biomech Eng 122: 15

14. Schneiderbauer M, Frigg A, Valderrabano V et al. (2005) Arthroskopische Befunde bei der chronischen Sprunggelenksinstabilität. Arthroskopie 18: 104-111

15. Tohyama H, Yasuda K, Ohkoshi Y et al. (2003) Anterior drawer test for acute anterior talofibular ligament injuries of the ankle: how much load should be applied during the test? Am J Sports Med 31: 226-232

16. Frost SC, Amendola A (1999) Is stress radiography necessary in the diagnosis of acute or chronic ankle instability? Clin J Sport Med 9: 4045

17. Frigg $A$, Magerkurth $O$, Valderrabano $V$ et al. (2007) The effect of osseous ankle configuration on chronic ankle instability. Br J Sports Med 41: 420 424
18. Olmsted LC, Carcia CR, Hertel J et al. (2002) Efficacy of the star excursion balance tests in detecting reach deficits in subjects with chronic ankle instability. J Athl Train 37: 501-506

19. Kuni B, Schmitt H (2004) Kraft und Propriozeption am Sprunggelenk bei Tänzern in der professionellen Ausbildung. Sportverletz Sportschaden 18: 15-21

20. Harrington KD (1979) Degenerative arthritis of the ankle secondary to long-standing lateral ligament instability. J Bone Joint Surg Am 61: 354-361

21. Lofvenberg R, Karrholm J, Lund B (1994) The outcome of nonoperated patients with chronic lateral instability of the ankle: a 20-year follow-up study. Foot Ankle Int 15: 165-169

22. Verhagen $E$, Beek $A$ van der, Twisk et al. (2004) The effect of a proprioceptive balance board training program for the prevention of ankle sprains: a prospective controlled trial. Am J Sports Med 32: 1385-1393

23. Hamilton WG, Thompson FM, Snow SW (1993) The modified Brostrom procedure for lateral ankle instability. Foot Ankle 14: 1-7

24. Pagenstert $G$, Valderrabano V, Hintermann B (2005) Lateral ankle ligaments reconstruction with free plantaris tendon graft. Tech Foot Ankle Surg 4: 104-112

25. Krips $\mathrm{R}$, Lehtonen $\mathrm{H}$, Halasi $T$ et al. (2002) Factors contribution to chronic ankle instability: kinesthesia and joint position sense. Am J Sports Med 30: 13-19 


\section{CME-Fragebogen}

Bitte beachten Sie:

- Antwortmöglichkeit nur online unter: CME.springer.de

- Die Frage-Antwort-Kombinationen werden online individuell zusammengestellt.

- Es ist immer nur eine Antwort möglich.
Hinweis für Leser aus Österreich

Gemäß dem Diplom-Fortbildungs-Programm (DFP) der Österreichischen Ärztekammer werden die auf CME.springer.de erworbenen CME-Punkte hierfür 1:1 als fachspezifische Fortbildung anerkannt.
Welches Band wird bei einer akuten Distorsion am oberen Sprunggelenk am häufigsten geschädigt?

$\square$ Ligamentum tibiofibulare anterius.

$\square$ Springligament.

$\square$ Ligamentum fibulotalare anterius.

$\square$ Tiefer Anteil vom Ligamentum tibiotalare anterius.

$\square$ Ligamentum tibionaviculare.

Der Deltabandapparat spielt bei der Stabilisierung des oberen Sprunggelenkes eine herausragende Rolle. Er limitiert vor allem die:

$\square$ Supination.

$\square$ Inversion vor allem in Plantarflexion und neutraler Flexionsstellung.

$\square$ Inversion in der Dorsalextension.

$\square$ Talusabduktion und Tibiaaußenrotation.

$\square$ Vorfußadduktion.

Was ist der Hauptbestandteil der Trockenmasse von

Ligamenten?

$\square$ Proteoglykane.

$\square$ Elastin.

$\square$ Fibrilläres Kollagen.

$\square$ Keratinsulfat.

$\square$ Aggrecan.

Was ist der wichtigste Risikofaktor für das Erleiden einer Distorsion am oberen Sprunggelenk?

$\square$ Positive Familienanamnese.

$\square$ Eine bereits durchgemachte Distorsion. $\square$ Teilnahme an Orientierungsläufen.

$\square$ Pes-planovalgus-Fehlstellung.

$\square$ Peronealsehnenluxation.

Man unterscheidet zwischen lateraler und medialer Sprunggelenkinstabilität. Wie wird die Kombination beider bezeichnet?

$\square$ Funktionelle Instabilität.

$\square$ Rotationsinstabilität.

$\square$ Posturale Instabilität.

$\square$ Translationsinstabilität.

$\square$ Neuromuskuläre Instabilität.

Die chronische Sprunggelenkinstabilität kann anhand des Pathomechanismus in eine mechanische und eine funktionelle Instabilität unterteilt werden. Welcher der folgenden Befunde spricht für eine funktionelle Instabilität?

$\square$ Hochgradige Instabilität im Drawer-Test.

$\square$ Druckdolenz im Bereich des Ligamentum deltoideum.

$\square$ Laterale Aufklappbarkeit im Talar-Tilt-Test.

$\square$ Schlechtes Ergebnis beim Star-excursion-balance-Test

$\square$ Positives Single-heel-riseZeichen.

Was ist der Goldstandard in der Diagnostik einer chronischen Instabilität des oberen Sprunggelenks?

$\square$ Stressradiographie.

$\square$ SPECT-CT.

$\square$ MRI.

Arthroskopie.

$\square$ Sonographie.
Welche Begleitpathologie wird häufig bei chronischen Instabilitäten arthroskopisch gefunden?

$\square$ Chondrale Läsion.

$\square$ Gelenkchromatose.

$\square$ Villonoduläre Synovitis.

$\square$ Fibulafraktur.

$\square$ Kristallarthropathie.

Ein 14-jähriger Basketballer stellt sich in der Ambulanz vor, nachdem er beim Training umgeknickt sei (anamnestisch erstmaliges Umknicken). Die klinische Untersuchung zeigt eine deutliche Schwellung im lateralen OSG mit anterolateralem Druckschmerz; kein Bluterguss sichtbar. Röntgen (OSG, 2 Ebenen) unauffällig.

Wie ist das weitere Prozedere?

$\square$ Wiedervorstellung zur diagnostischen Arthroskopie.

$\square$ Durchführung einer Stressradiographie.

$\square$ Bandrekonstruktion nach Brostrom-Gould.

$\square$ Abschwellende Maßnahmen.

$\square$ Abschwellende Maßnahmen und frühfunktionelle Physiotherapie.

Ein 20-jähriger Mann, der in der Freizeit Orientierungsläufe macht, stellt sich in der Sprechstunde vor. Er habe verstärkt Schmerzen und ein Instabilitätsgefühl im linken Sprunggelenk, besonders beim Laufen im

Wald. Er sei schon mehrmals umgeknickt, vor allem am Ende einer Trainingseinheit.
Die klinische Untersuchung zeigt: Schubladentest $2^{\circ}$ positiv, medialer und lateraler Aufklappbarkeitstest $2^{\circ}$ positiv, Single-heel-rise-Zeichen negativ, Rückfuß-Alignement $5^{\circ}$ valgus, pathologischer Star-excursionbalance-Test. Eine intensive Physiotherapie und Stabilisierung mit einem Brace brachte bisher keine Besserung. Wie ist das weitere Prozedere?

$\square$ Medial-sliding-calcaneusOsteotomie mit Rekonstruktion der Tibialis-posteriorSehne nach Cobb.

$\square$ Tenodese nach Watson-Jones.

$\square$ Arthroskopie, ggf. anschlieBende direkte Bandrekonstruktion nach BrostromGould.

$\square$ Dwyer-Osteotomie mit Peroneus-longus-auf-BrevisSehnenstransfer.

$\square$ Konservative Weiterbehandlung.

Diese Fortbildungseinheit ist 12 Monate auf CME.springer.de verfügbar.

Den genauen Einsendeschluss erfahren Sie unter

CME.springer.de 